\title{
Voix narratrices et (dé)canonisation de la littérature chez Ahmed Zitouni
}

\author{
Monique Manopoulos \\ The University of Memphis
}

A hmed Zitouni, né en Algérie et vivant à Aix-en-Provence depuis 1973, est un auteur qui produit régulièrement des textes depuis la parution de son premier roman en 1983. Ses textes offrent aux lecteurs un monde de personnages, de narrateurs et d'écriture très complexe qui n'est pas dénué d'humour malgré le sérieux des propos. Ce qui frappe le plus dans les écrits d'Ahmed Zitouni est la présence narrative de plusieurs voix/voies que ce soit dans son premier roman, Avec du Sang déshonoré d'encre à leurs mains, ou son dernier, Une difficile fin de moi (publié en 1998). Ces voix qui constituent la trame des récits de tous ses textes vont au-delà d'une stratégie narrative originale. En fait, je pense qu'elles sont à la base d'une déconstruction des concepts conventionnels de narrateurs diégétiques et extra-diégétiques. Effectivement, bien que les utilisant au niveau du récit, il les inclue et simultanément les dépasse pour atteindre des possibilités de signifiance qui édifient une subtile déconstruction des conventions narratives. Les diverses voix/voies sont à la fois à l'intérieur du récit et en marge de celui-ci; et ce faisant Ahmed Zitouni crée en quelque sorte un panoptisme ${ }^{1}$ décentré où chaque voix/voie surveille l'autre/les autres. Cette caractéristique qui constitue le coeur des écrits d'Ahmed Zitouni permet de produire un commentaire sous-jacent et constant sur la convention littéraire comme agent de pouvoir et par conséquent sur le concept de canon littéraire.

Cette constance de parcours périphériques existe non seulement dans les écrits de l'auteur lui-même mais également dans ceux de ses protagonistes dont les voix passent par leurs propres écrits. Par exemple, Attilah Fakir, le personnage d'Attilah Fakir le roman, a écrit un roman intitulé La balade du bicot, le pendu, personnage de La veuve et le pendu, a écrit un journal. Sont ainsi créés des déplacements et décentrements continuels de perspectives narratives, des écrits dans les écrits et à leur périphérie.

Je pense donc qu'il existe un parallèle entre la position périphérique des personnages dans les récits, qui constitue un panoptisme décentré, et la position périphérique des stratégies narratives qui constitue un décentrement du canon littéraire. Les personnages d'Ahmed Zitouni sont tous des marginaux solitaires, et ce de façons diverses (comme nous 
le verrons plus tard), qui effectuent des parcours périphériques physiquement et/ou mentalement. Par exemple, dans Avec du Sang déshonoré d'encre à leurs mains, le narrateur effectue un parcours isolé à travers les faits divers de plusieurs journaux qu'il habite, à la recherche de son ami M.X; et dans Attilah Fakir, Attilah effectue d'une part des randonnées journalières à travers Aix-en-Provence et d'autre part a écrit un ouvrage intitulé La Balade du bicot. Lors de leurs péripéties, les personnages sont à la fois surveillés-puisque dans la structure panoptique le pouvoir est central et ils sont dans les marges en tant qu'étrangers - et surveillants puisqu'ils observent et déstabilisent les mécanismes de surveillance de par leur marginalité et de par le fait que, se sachant surveillés, ils dé-jouent le mécanisme. Par exemple, dans Aimez-vous Brahim, Bensaâdi Kadda/Brahim adopte un discours et une position de colon devant les surveillants de l'asile notamment en traitant le narrateur de "taré de bicot", mais la nuit il adopte un discours tout empreint de camaraderie envers ce même narrateur. Il décentre ainsi le panoptisme existant en le court-circuitant; ce qui rappelle cette réflexion de Derrida:

Parce que le maître ne possède pas en propre, naturellement, ce qu'il appelle pourtant sa langue; parce que quoi qu'il veuille ou fasse, il ne peut entretenir avec elle des rapports de propriété ou d'identité naturels, nationaux, congénitaux, ontologiques; parce qu'il ne peut accréditer et dire cette appropriation qu'au cours d'un procès non naturel de constructions politico-phantasmiques; parce que la langue n'est pas son bien naturel, par cela même il peut historiquement, à travers le viol d'une usurpation culturelle, c'est-à-dire toujours d'essence coloniale, feindre de se l'appropier pour l'imposer comme «la sienne». ${ }^{2}$

Cette citation se rapportant à l'usurpation d'une langue par le maître-colon, illustre bien le phénomène de déconstruction par renversements de structures de pouvoir en se jouant de ces dernières, tout en les utilisant. Brahim, le "bicot", usurpe la langue du "maître"--ce qui renvoie à l'usurpation de la langue par le "maître" - et ce faisant met la possession/usurpation de la langue, dite naturelle, en abîme. Ainsi la boucle possesseur/usurpateur de la langue du pouvoir déconstruit la notion de pouvoir central.

Ce qui permet d'affirmer que le parallèle entre déconstruction des voix/voies narratrices et (dé)canonisation est primordial réside dans le fait que le roman Attilah Fakir est le symbole d'une attaque du pouvoir canonique d'une émission littéraire française bien connue "Apostrophes". Comme le précise la femme d'Attilah: "Tu es un écrivain, maintenant. C'est ce que tu as toujours voulu, n'est-ce pas! Et même un grand écrivain, puisque la télévision va te rendre célèbre!" (111). Dans cette remarque se trouve résumé tout le concept de canon littéraire. Un écrivain est consacré grand écrivain seulement s'il est estampillé par le pouvoir littéraire institutionalisé du moment, et dont "Apostrophes" est le symbole dans l'inconscient collectif français. Attilah, l'écrivain, est donc invité à cette émission, qui fait en France figure d'institution, mais durant celle-ci il reste complètement silencieux. Il prend ainsi le rôle de surveillé/surveillant puisqu'il participe sans participer

\section{LittéRéalité 52}


(décentrement et renversement; canonisation et décanonisation.

Ce décentrement de pouvoir se manifeste par le biais de diverses stratégies narratives qui forment des mouvements circulaires à travers, simultanément, la narration périphérique des divers personnages et la présence de plusieurs voix extra-diégétiques et diégétiques. Ces caractéristiques produisent alors non pas une trame de lecture mais un réseau de jeux de possibilités dont la seule affirmation est le décentrement de tout canon.

\section{Panoptisme initial/panoptisme décentré}

Tous les personnages principaux des différents écrits sont des péripatéticiens ${ }^{3}$, physiquement et/ou mentalement, au sens aristotélicien et au sens littéral. Leurs diverses balades s'effectuent à travers des villes et à travers des pensées - Attilah Fakir, Aimezvous Brahim —; d'autres à travers l'écrit — La veuve et le pendu, Avec du sang déshonoré d'encre à leurs mains, Amour, sévices et morgue - et finalement à travers le temps et l'espace - Une difficile fin de moi. Ces mouvements périphériques se multiplient euxmêmes car tous les personnages se meuvent en déplacements panoptiques en leur intérieur comme par exemple dans Aimez-vous Brahim:

Bizarre cette manière de se déplacer? J'essayais de comprendre le pourquoi de ce zigzag persistant. (53)

Ce salaud patrouille en permanence dans la chambre. (59)

Brahim marchait toujours. S'approchait. Repartait. N'en finissait pas de me contourner. (114)

Il se tut et continua de marcher. Toujours en faisant des cercles autour de moi. (116)

Ces mouvements circulaires/périphériques créent des déplacements et par conséquent glissements 4 physiques, sémantiques et symboliques qui permettent aux personnages d'échapper aux mécanismes de contrôle initiaux et de les décentrer par le biais de spirales dont il est difficile d'extraire le premier mouvement.

Les personnages principaux des divers livres sont initialement dans une position marginalisée par rapport aux formes de pouvoir quelles qu'elles soient et sont donc surveillés puisqu'à l'extérieur. Mais tous les personnages finissent par dépasser cette position limitée en la subvertissant sans toutefois la nier mais en la comprenant et la décentrant. Ils prennent ainsi un avantage par rapport au pouvoir qu'il ne peuvent supprimer, comme il est indiqué dans plusieurs passages de Aimez-vous Brahim: "De mon poste d'observation, je contrôle les allées et venues" (28); "Je l'observe. Et plus je l'observe, plus je constate que c'est un simulateur." (51)

Dans Avec du sang déshonoré d'encre à leurs mains, le narrateur est surveillé par le pouvoir central de ceux qu'il appelle "les concierges", c'est-à-dire les journalistes, car il habite les colonnes des faits divers en tant que victime d'une bavure. De plus, étant 
d'origine maghrébine, il est automatiquement surveillé par tout un réseau de contôle. Il est ainsi en double position périphérique mais cette double périphérie permet également un premier décentrage puisqu'il est à la périphérie de deux points centraux qui ne coïncident pas. Un deuxième décentrage vient du fait que malgré cette position statique, en apparence à la merci du pouvoir, des journalisteset de la police, le narrateur échappe à leur contrôle en vivant après sa mort à travers le livre et dans les faits-divers, d'une part, et d'autre part en décidant de parcourir les faits divers de tous les journaux à la recherche de M.X alias Abderhamane alias Impermastic. Ce M.X occupe lui aussi une position de départ doublement surveillée. Il est maghrébin et il est surveillé par le narrateur qui le cherche. Il échappe aussi aux deux pouvoirs centraux ainsi établis. Il échappe au pouvoir administratif en "se faisant suicider" par la police française pour avoir tué un maghrébin, Bouzid, et il se dérobe aux recherches du narrateur. En outre, M.X acquiert une position centrale sporadique en étant le narrateur de certains passages. Grâce aux doubles décentrements du narrateur et de M.X chacun devient simultanément centre et périphérie de l'autre et des autres.

Dans Atillah Fakir, Attilah se trouve dans une position panoptique des plus complexes. D'une part il est automatiquement surveillé par le pouvoir de la police française-puisqu'immigré - et d'autre part il dépend du pouvoir central de la littérature française puisqu'il est écrivain invité de l'émission/institution "Apostrophes". En outre, il est en position centrale par rapport aux deux cultures qui le délimitent, la française et la maghrébine. Or Attilah déjoue toutes ces structures centralisatrices grâce à des stratagèmes divers. Il effectue des balades journalières et solitaires à travers les méandres de la ville d'Aix-en-Provence au cours desquelles il déstabilise les symboles des pouvoirs administratifs, par exemple en renommant toutes les rues à l'aide de noms de marginaux. De plus, il accomplit cela en faisant précéder les noms du titre de "Hadj" normalement réservé à ceux qui, dans le passé, ont effectué un pélerinage à la Mecque. Un autre mouvement périphérique se trouve dans le fait que lorsqu'Attilah se trouve dans l'antre du canon littéraire symbolisé par l'émission "Apostrophes", il ne se livre pas à cette institution et la décentre en ne prononçant pas un seul mot, récupérant ainsi le pouvoir litéraire du non-dit au lieu de la consécration canonique qui récupère toute expression individuelle. Une autre dimension de ce mouvement périphérique se trouve dans les rituels qui transforment le réel d'Attilah par un procédé d'alchimie fakirienne qui se sert d'objets dont la symbolique est unique; par exemple les oeuvres des Hadj sont situées à la suite, dans l'ordre, de chaussures (migration), d'une tenue de plongée (étrangeté/exploration) et de bocaux contenant toutes les épices du Maghreb (magie/orientalisme). Tout cela pour affirmer; "je m'appelle Moi" (89), alors qu'Attilah veut dire "celui qui détruit tout sur son passage" (identité conventionnelle). Enfin, le titre du livre écrit par Attilah Fakir, La Balade du bicot contient à lui seul toute la symbolique des divers mouvements périphériques.

Dans La Veuve et le pendu, la position du personnage principal, le pendu, est simultanément périphérique - il regarde sa femme lire son journal alors qu'il est mort - 
et centrale - il est le narrateur du récit et de son journal lu par sa femme. Par conséquent il est surveillé - son journal et sa dépouille - et il surveille puisque son récit nous permet d'observer sa femme en train de le surveiller.

Les personnages principaux de Aimez-vous Brahim, le narrateur et Bensaâdi Kadda/Brahim, sont physiquement et mentalement dans un système panoptique à l'intérieur d'un asile d'aliénés, donc en dehors de la vie normale et en périphérie de la ville. A partir de cette position dans l'anneau panoptique, ils décentrent physiquement les pouvoirs. D'une part, ils effectuent une descente sur le centre ville avec toute une "armée" d'aliénés. D'autre part, ils "se font suicider" par deux policiers; ce qui met un terme (physique) à leur escapade. Ainsi, le pouvoir marginalisé/central représenté par le narrateur qui voulait exercer son pouvoir en tuant Bensaâdi Kadda, se trouve décentré. Le pouvoir central représenté par les policiers se trouve décentré car les deux protagonistes avaient en fait monté une opération suicide. Finalement, le pouvoir marginalisé représenté par Bensaâdi $\mathrm{Kadda} / \mathrm{Brahim}$ est décentré puisque son dernier acte lui confère le pouvoir sur la narrateur en lui volant son meurtre, et sur les policiers en les obligeant à le tuer; ce qui était le but qu'il recherchait.

Dans Amour, sévices et morgue, il s'agit d'un maghrébin, Malik, surveillé par le narrateur qui s'intéresse à son histoire après l'avoir lue dans les journaux, et par les pouvoirs administratifs puisqu'il est "étranger". Il décentre le pouvoir en tuant ses trois enfants, sa belle-mère, sa belle-soeur et lui-même. De plus, en épargnant sa femme française, il la place dans une position démunie de tout pouvoir de vengeance.

En ce qui concerne le dernier livre, Une Difficile fin de moi, le narrateur est simultanément surveillé en tant que Maghrébin et gréviste de la faim mais surveillant en tant que médecin et narrateur maître du récit et de la fin de sa vie.

Ainsi, outre les valeurs significatives des mouvements périphériques observés, une autre dimension se rajoute par le biais de l'évocation du concept de nomadisme - cliché exotique du Maghreb, mouvement du migrant. Il s'agit de la déconstruction de l'identité fixe, c'est-à-dire de l'identité administrative donnée par les pouvoirs centraux.

\section{Voix extra-diégétiques \\ Intertextes}

Les intertextes créent un texte en marge, donc périphérique qui fait miroir au mouvement décentreur de panoptisme et établit également des niveaux multiples. Dans Une difficile fin de moi et Amour, sévices et morgue, l'intertexte est constitué de citations d'auteurs et de grévistes de la faim de pays et époques différents qui marquent simultanément un centrage du récit et un texte circulatoire à part entière. Par exemple la récurrence de citations extraites de Un artiste de la faim de Kafka telle la suivante: "Il est certain, naturellement, que le temps des jeûneurs reviendrait un jour mais ce n'était pas une consolation pour les vivants." (23). Les autres citations directes viennent de grévistes de la faim à travers le temps et l'espace et de rapports médicaux authentiques sur la grève de 
la faim et leur nourriture forcée - torture - qui forment un autre récit circulaire:

J'étais entourée, refoulée sur la chaise. qui était inclinée vers l'arrière. Ils étaient une dizaine. Le docteur me força la bouche de façon à obtenir une poche et me tint pendant que les gardiennes y versaientt du liquide à la cuillère; (...) L'extrémité est enfoncée un jour dans une narine, le lendemain dans l'autre. La manoeuvre est très douloureuse... Les typans des oreilles semblent éclater, une horrible douleur s'étale dans la gorge et la poitrine.[...]." Témoignage de Mary Leigh, cité par Jean-Philippe Mangeon (d'après Rosen Andrew, Rise up Women) colloque sur «La grève de la faim le dérèglement du sacré». (89)

Cette citation renvoie d'intertexte à intertexte, multipliant par là même les voix narratrices.

En ce qui concerne Amour, sévices et morgue, le mouvement marginal circulaire se trouve dans la récurrence d'extraits d'articles de journaux authentiques relatant le crime de Malik, seul le prénom ayant été changé.

Dans Aimez-vous Brahim et Avec du Sang déshonoré d'encre à leurs mains, il s'agit d'allusions directes à des auteurs du canon français tel Rabelais et Verlaine - bien sûr le titre venant directement d'un poème de ce dernier:

Ces femmes! Dis les gaz, et l'horreur identique

Du mal toujours, du laid partout sur tes chemins,

Et dis l'Amour et dis encor la Politique

Avec du sang déshonoré d'encre à leurs mains. ${ }^{5}$

Il existe également un intertexte interne puisque l'on retrouve le Royal Bar comme étape de balade dans Attilah Fakir et dans Aimez-vous Brahim., et un personnage Krimo dans Avec du Sang déshonoré d'encre à leurs mains et Aimez-vous Brahim.

\section{Voix intra-diégétiques}

Les voix intra-diégétiques sont toujours composées de deux voix de base qui s'opposent mais se renversent continuellement puisqu'une ne prend pas prépondérance par rapport à l'autre. Toute opposition binaire est ainsi dépassée pour donner lieu à une périphérie faite de voix multiples et combinatoires à l'instar de la binarité de départ narrateur/M.X dans Avec du Sang déshonoré d'encre à leurs mains. Dans cet ouvrage la première binarité donne naissance à l'intérieur de la périphérie des faits divers à une prolifération de voix qui dialoguent entre-elles - le narrateur/José ou Luis ou Miguel/Hector Chareux/M.X ou Abderhamane ou Impermastic. On finit même par se demander s'il ne s'agit pas d'une seule voix multiple court-circuitée par le narrateur/simulateur si l'on en croit l'avertissement:

Avant toute incartade de dire, je tiens à préciser que le bougre qui s'est acharné à 
traverser les pages de ce livre en semi-clandestin de lits et ratures (l'expression est du sieur Duchamp), est loin d'être un quelconque personnage de fiction: un héros, comme l'habitude le fait dire si souvent! (9)

Etant donné que cet avertissement nous est donné par le narrateur, nous pouvons nous attendre à de la simulation et à du jeu. De plus, concernant la décanonisation, il est intéressant de remarquer "comme l'habitude le fait dire souvent".

Dans Une Difficile fin de moi, ces voix intra-diégétiques ont comme base la binarité dialogique: le narrateur et sa voix en tant que médecin:

- Ma parole! c'est l'hôpital qui se fout de la charité. Il fallait s'y attendre. Le revoilà. La morgue et la suffisance, en prime.

- Ongles secs et cassants. Perte de cheveux significative. Je le vois venir. Grand comme un gigot. Il veut me faire croire qu'il existe pour de vrai. Me la joue sur le registre du double, des hallucinations. M'entraîne vers des sentiers de folie. je le vois venir et m'en méfie. Je suis tout ce qu'il y a de plus lucide en ce monde, Ducon. Il ricane. (37)

A celles-ci s'ajoutent les dialogues avec les divers grévistes de la faim que ce soit Fathia, une femme en Algérie qui hante régulièrement l'esprit du narrateur (autre périphérie), Gerry Adams, membre de l'I.R.A, ou Mary Leigh, une sufragette britannique, Agrippine l'Aînée, femme de Germanicus ou des sans-papiers. Toutes ces voix créent des voies périphériques au récit de base et par conséquent multiples.

Dans Attilah Fakir, la binarité de base Attilah/la voix constitue à elle seule un dialogue qui démultiplie puisque durant tout le récit nous avons l'impression que c'est Attilah qui crée cette dernière mais à la fin du récit un renversement inattendu révèle que c'est également la voix qui contrôle le sort d'Attilah: "Mais j'avais perdu Attilah Fakir. Nul regret pour sa carcasse et ses coups de gueule. Je l'avais amené où il devait finir, où je finissais avec lui. Seul." (250). Il faut rajouter à cela un dialogue tacite avec les Hadj qui constitue une décanonisation du canon islamique, puisqu'Attilah donne au terme de "Hadj" une définition tout à fait personnelle:

...Et d'autres monuments de dignité debout, des Hadj qui surent cracher au visage de leurs charitables bienfaiteurs, quelles que furent leurs intentions. La Justice, avec une majuscule aussi grande que l'oppression, voilà ce qui manque dans l'infini connerie de la comédie humaine. (109)

Ces Hadj comptent parmi eux des personnes aussi variées que: Kubrick, Fanon, Hegel, Ravel, Makhno - un anarchiste russe - Memmi, Faulkner, Artaud, etc. Certains sont marginaux, d'autres centraux mais on été à un moment marginaux. Attilah recrée ainsi une autre forme de canon mais par le biais d'un glissement sémantique car il applique un terme 
religieux aux arts. Ainsi il ne nie pas le canon mais le subvertit en le décentrant. Attilah se décentre par rapport au pouvoir français en procédant à l'intrônisation des Hadj mais simultanément il se décentre par rapport au pouvoir maghrébin en subvertissant cette notion de Hadj pour désigner ceux qui selon lui démontrent un engagement envers l'Humanité:

... où sommeille en ce moment une faune cosmopolite: deux Argentins désespérément fous, un Mexicain alcoolique, un nobelisable du Nigéria, trois Américains (dont l'inévitable suicidé de service) quelques apatrides... dont deux dits d'expression française, une cohorte de dissidents divers, ainsi qu'une flopée de génies de la langue... (36)

Une autre voix/voie qui parcourt les écrits d'Ahmed Zitouni réside dans le fait que ses personnages écrivent des lettres aux administrations françaises. Ces lettres sont un symbole de l'acte d'écriture décentriste. Etant donné que l'identité--par-là également l'existence-- officielle selon les pouvoirs administratifs doit étre sanctionnée par des papiers officiels, la réappropriation du pouvoir et le décentrement du panoptisme sont sanctionnés par l'écriture littéraire de papiers non-officiels mais envoyés à des bureaux officiels tels celui des allocations familiales, l'administration pénitentière et autres. De plus, lorsque par exemple Attilah adresse sa lettre aux allocations familiales à "Messieurs de la S.S. à régulateur de chair à canon et d'instigateurs de copulations sans plaisir" (40), il subvertit l'identité acceptée/officielle de cet organisme. Il en est de même lorsqu'il termine sa lettre de demande d'emploi au directeur de la prison en ces termes: "En prenant congé de votre peu avenante personne et avec tout le dédain que je dois à votre ignoble profession, recevez mes distantes et administratives salutations." (45) Il s'agit de décentrement puisqu'il subvertit les formules de politesses officielles en gardant la formule fixe/acceptée tout en lui donnant une tournure sémantique tout à fait personnelle. Ces administrations représentent le pouvoir panoptique de contrôle des immigrés qui doivent constamment affronter l'administration française afin d'avoir une identité, donc une existence, reconnue et qui constitue un système de surveillance. Les lettres litéraires envoyées à ces systèmes de contrôle décentrent ce système et au niveau litéraire, elles symbolisent la (dé)canonisation de l'écriture française par un "immigré français".

Les caractéristiques péripatéticiennes des récits d'Ahmed Zitouni créent un réseau de décentrements multiples qui ont pour base la canon litéraire car le canon est fixe, la (dé)canonisation est mouvante, le pouvoir est fixe, la marge est mouvante. Le terme canon littéraire évoque les différentes définitions trouvées dans un dictionnaire, que ce soit dans le domaine religieux ou philosophique, c'est-à-dire "des règles, une norme", donc une fixité qui sert de modèle, donc une mort de la parole, de l'être. Cette connotation de mort est soulignée dans le concept de canonisation des saints, or Ahmed Zitouni décentre subtilement ces caractéristiques en créant des narrateurs qui écrivent et parlent alors qu'ils sont déjà morts, subvertissant ainsi toute notion de codification inamovible. De plus, les mouvements périphériques décentreurs amènent les lecteurs dans les marges et les placent 
dans une position de surveillés/surveillants qui échappent à tout contrôle stabilisateur. En conséquence, ils répercutent à l'extérieur du récit le mouvement à l'intérieur du récit où chacun est l'autre.

Enfin, il est important de noter que l'itinéraire littéraire d'Ahmed Zitouni fait écho à l'itinéraire de ses personnages puisque ses textes ne suivent pas une formule stable, ils évoluent constamment tout en conservant les éléments décentreurs. Il crée ainsi une (dé)canonisation de sa propre écriture.

Notes

1 Notion élaborée en particulier par Michel Foucault dans Surveiller et punir (Paris: Gallimard, 1975) et décrite ainsi: "Le Panoptisme de Bentham est la figure architecturale de cette composition. On en connaît le principe: à la périphérie un bâtiment en anneau; au centre une tour; celle-ci est percée de larges fenêtres qui ouvrent sur la face intérieure de l'anneau (...). Il suffit alors de placer un surveillant dans la tour centrale, et dans chaque cellule d'enfermer un fou, un malade, un comdamné, un ouvrier ou un écolier." (p.201-2).

2 Jacques Derrida. Le Monolinguisme de l'autre. (Paris: Galilée, 1996). p.45.

3 de peripateteuein: se promener, parce qu'Aristote enseignait en se promenant. Armand Cuvillier. Nouveau vocabulaire philosophique. (Paris: Colin, 1970).

${ }^{4}$ Cf. Roland Barthes. Degré zéro de l'écriture. (Paris: Seuil, 1953).

5 Paul Verlaine. "Sagesse, III". La bonne chanson. (Paris: le livre de Poche, 1963) 


\section{Bibliographie}

Barthes, Roland. Degré zéro de l'écriture. Paris: Seuil, 1953.

Cuvillier, Armand. Nouveau vocabulaire philosophique. Paris: Colin, 1970.

Derrida, Jacques. Le Monolinguisme de l'autre. Paris: Galilée, 1976.

Foucault, Michel. Surveiller et Punir. Paris: Gallimard, 1975.

Verlaine, Paul. La bonne chanson. Paris: le livre de Poche, 1963.

Zitouni, Ahmed. Avec du sang déshonoré d'encre à leurs mains. Paris: Laffont, 1983. . Aimez-vous Brahim? Paris: Belfond, 1986.

. Amour, sévices et morgue. Paris: Parc, 1998.

- Attilah Fakir. Paris: Souffles, 1987.

. La Veuve et le pendu. Paris: Manya, 1993.

. Une Difficile fin de moi. Paris: Le Cherche Midi, 1998. 